\title{
Wideband Tapered Slot Antenna for D-Band Applications
}

\author{
Dong, Yunfeng; Turhaner, Arsen; Zhurbenko, Vitaliy; Johansen, Tom Keinicke
}

\section{Published in:}

Proceedings of 2020 14th European Conference on Antennas and Propagation

Link to article, DOI:

10.23919/EuCAP48036.2020.9135911

Publication date:

2020

Document Version

Peer reviewed version

Link back to DTU Orbit

Citation (APA):

Dong, Y., Turhaner, A., Zhurbenko, V., \& Johansen, T. K. (2020). Wideband Tapered Slot Antenna for D-Band Applications. In Proceedings of 2020 14th European Conference on Antennas and Propagation (pp. 1-4). IEEE. https://doi.org/10.23919/EuCAP48036.2020.9135911

\section{General rights}

Copyright and moral rights for the publications made accessible in the public portal are retained by the authors and/or other copyright owners and it is a condition of accessing publications that users recognise and abide by the legal requirements associated with these rights.

- Users may download and print one copy of any publication from the public portal for the purpose of private study or research.

- You may not further distribute the material or use it for any profit-making activity or commercial gain

- You may freely distribute the URL identifying the publication in the public portal 


\title{
Wideband Tapered Slot Antenna for D-Band Applications
}

\author{
Yunfeng Dong*1, Arsen Turhaner*2, Vitaliy Zhurbenko*3, and Tom K. Johansen*4 \\ *Electromagnetic Systems, Department of Electrical Engineering \\ Technical University of Denmark \\ DK-2800 Kgs. Lyngby, Denmark \\ $\left\{1\right.$ yundon, ${ }^{2}$ aturh, ${ }^{3}$ vz, ${ }^{4}$ tkj $\} @$ elektro.dtu.dk
}

\begin{abstract}
This paper presents a wideband tapered slot antenna for D-band (110-170 GHz) applications. The antenna is fed by a substrate integrated waveguide (SIW) and achieves a simulated gain of $11.1 \mathrm{dBi}$ at $170 \mathrm{GHz}$. The in-band gain variation is less than $3 \mathrm{~dB}$. The designed SIW is based on a thin aluminium nitride (AIN) substrate with hollow plated vias working as the vertical conductor walls. The substrate is extended and inserted into a tapered slot antenna. The concept of designing SIW-fed tapered slot antenna is described in detail. The assembly structure is illustrated and the antenna radiation pattern is shown. The proposed SIW-fed tapered slot antenna also provides a wideband matching at $D$-band. The simulated return loss remains better than $18 \mathrm{~dB}$ from $110 \mathrm{GHz}$ to $170 \mathrm{GHz}$ which corresponds to a bandwidth of $43 \%$ at $140 \mathrm{GHz}$.
\end{abstract}

Index Terms-D-band, substrate integrated waveguide (SIW), tapered slot antenna, wideband.

\section{INTRODUCTION}

With the increasing demand worldwide for high-speed wireless communication, high-resolution imaging, remote sensing, and security applications, millimeter-wave frequency bands above $100 \mathrm{GHz}$ have acquired enormous attention in recent years [1]-[4]. D-band covers the frequencies from $110 \mathrm{GHz}$ to $170 \mathrm{GHz}$. It becomes attractive due to low atmospheric loss and large bandwidth [5]. Different types of wideband antennas have been investigated for wireless point-to-point communications as well as system integration and packaging at millimeter-wave frequencies [6]-[9]. The challenge for designing such antennas is to achieve a high gain and at the same time a wideband impedance matching. Besides, the in-band gain uniformity needs to be taken into account for transmitting and receiving signals around the same power level at different frequencies.

Fig. 1 shows the overview of the proposed wideband tapered slot antenna for D-band applications. A substrate integrated waveguide (SIW) with an extension on the substrate is inserted into the gap guiding electromagnetic waves from the SIW to the tapered slot antenna and radiating into free space. In this way, the SIW works as the feed of the antenna and makes it compatible for connecting to other circuits as well as components. Compared with conventional rectangular waveguides, SIWs are more compact and versatile especially at millimeterwave frequencies. The dimensions of the waveguide can be downscaled by a factor of $\sqrt{\epsilon_{r}}$ in which $\epsilon_{r}$ represents the relative permittivity of the dielectric substrate. Besides, SIW

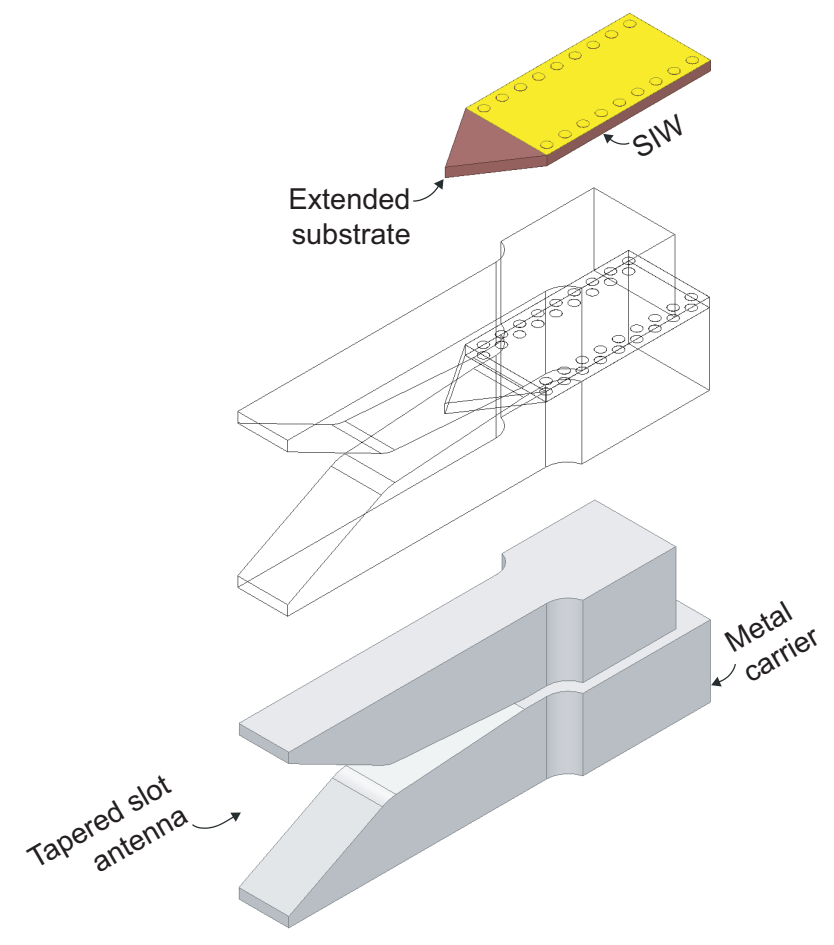

Fig. 1. Overview of the proposed SIW-fed wideband tapered slot antenna for D-band applications.

is easier to be integrated with the circuits at such high frequencies since they mainly rely on planar structures due to the fabrication processes. The transitions between SIWs and planar transmission lines used for the circuits at D-band can be found in the literature [10], [11].

In this work, a SIW-fed wideband tapered slot antenna for D-band applications is designed and analyzed by using the full-wave electromagnetic simulator from Ansys. In order to validate the concept, a thin aluminium nitride (AlN) substrate is used for designing the SIW while it can be reconfigured for other dielectric materials such as gallium arsenide (GaAs) or indium phosphide (InP). In Section II, the designs of the SIW with extended substrate and the tapered slot antenna are described in detail. The dimensions are shown and the fundamentals are explained. Besides, the assembly structure of the proposed antenna is demonstrated and the potential 


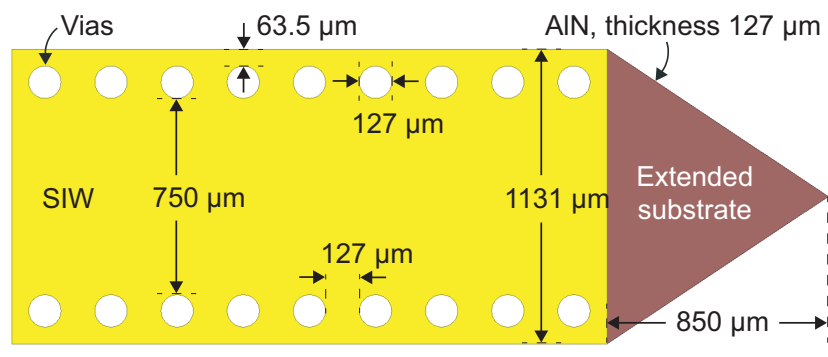

Fig. 2. SIW with extended AlN substrate.

way of building an antenna array is discussed. In Section III, the simulated scattering parameters, gain, radiation pattern of the proposed SIW-fed wideband tapered slot antenna are presented.

\section{Design Procedue of SIW-Fed Tapered Slot ANTENNA}

\section{A. SIW with Extended Substrate}

Conventional rectangular waveguides have both horizontal and vertical conductor walls forming an air-filled rectangular tube. At millimeter-wave frequencies, SIWs are normally based on thin dielectric substrates due to the downscaled wavelength. Under this circumstance, the top and bottom metal layers work as the horizontal conductor walls while the vertical conductor walls can be realized by adding vias going through the substrate. As the feed of the antenna, a SIW is designed by using an AlN substrate with hollow plated vias. The dissipation factor $(\tan \delta)$ and $\epsilon_{r}$ of the AlN substrate are 0.001 and 8.6, respectively. At the end of the SIW, the AlN substrate is extended while its width is reduced gradually forming a triangle termination. The extended substrate helps guiding electromagnetic waves smoothly from the SIW to the tapered slot antenna. The details of the designed SIW is illustrated in Fig. 2.

The material of the top and bottom conductor layers is gold and it has a thickness of $1.2 \mu \mathrm{m}$. The vias are located as close to each other as possible in order to prevent the wave leakage from the SIW. By taking into account the requirements of the fabrication processes, both the diameter of the vias and the gaps between the vias need to be kept more than the thickness of the substrate which is $127 \mu \mathrm{m}$ in this case. Fig. 3 shows the simulated scattering parameters of the designed SIW at D-band. The extension of the AIN substrate is excluded from the SIW simulation structure. The return loss remains better than $27 \mathrm{~dB}$ from $110 \mathrm{GHz}$ to $170 \mathrm{GHz}$ with an associated insertion loss of $0.3 \mathrm{~dB}$.

\section{B. Tapered Slot Antenna}

Fig. 4 demonstrates the proposed SIW-fed wideband tapered slot antenna. When the wave propagation is dominated by the $\mathrm{TE}_{10}$ mode in the designed SIW, the electric fields exist vertically between the gold conductor layers. As for the tapered slot antenna, the electric waves are excited in the small gap at the end of the slot and guided along the tapered

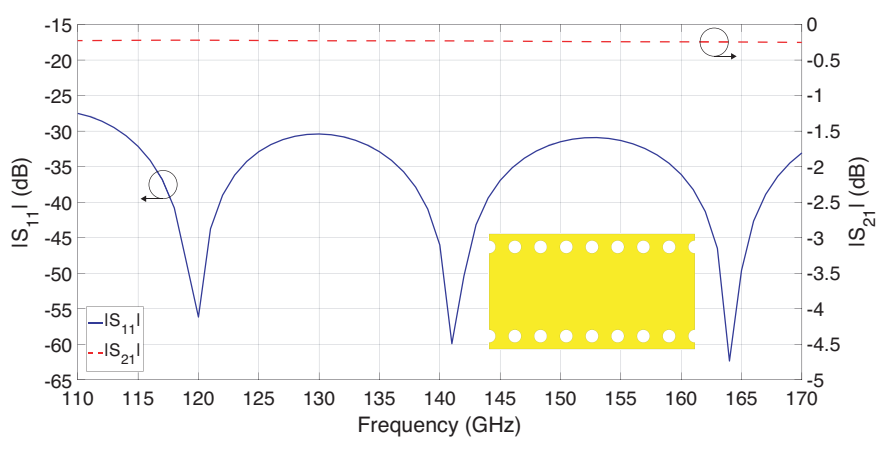

Fig. 3. Simulation results of the designed SIW.

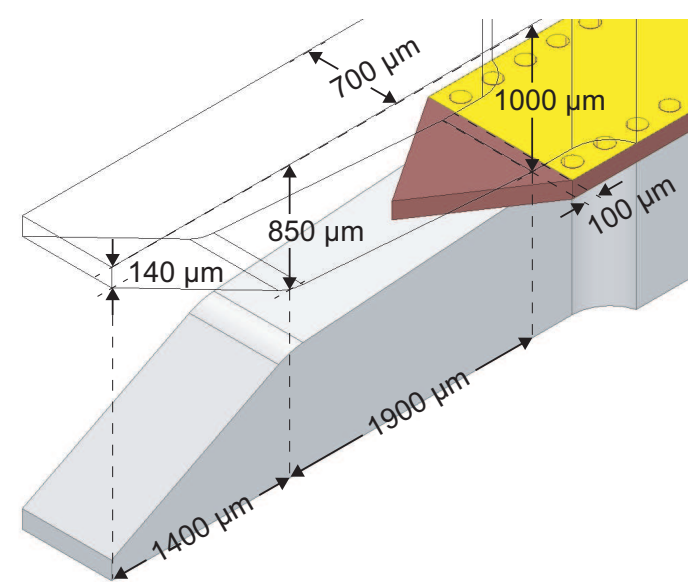

Fig. 4. Wideband tapered slot antenna.

structure. In order to support a wideband radiation, the length and height of the tapered slot are optimized by three stages. At the beginning, the AlN substrate is inserted into the gap and the slot is extended $100 \mu \mathrm{m}$. After that, the gap enlarges vertically while the AIN substrate is terminated gradually. The length of the second and third stages are $1900 \mu \mathrm{m}$ and $1400 \mu \mathrm{m}$, respectively. The connections between the second and third stages are also rounded preventing the possible reflections. The aperture width of the tapered slot antenna is $700 \mu \mathrm{m}$ and the height is comparable with the free-space wavelengths at D-band.

\section{Assembly Structure and Antenna Array}

The assembly strucrure of the proposed SIW-fed wideband tapered slot antenna is shown in Fig. 5. The tapered slot is made by aluminium block and divided into two identical parts for easier fabrication and alignment. The bottom part is connected to a metal carrier which contains an alignment slot with a thickness of $127 \mu \mathrm{m}$ guiding the SIW to the correct position. During the assembly process, the SIW with extended AlN substrate is aligned with the bottom metal carrier at the beginning. The rest half of the tapered slot is fixed from the top afterwards forming a clamping structure. Two guide pins and two screws are used for accurate assembly.

When higher directivity is required by the system or beamforming technique is involved, it is also possible to 

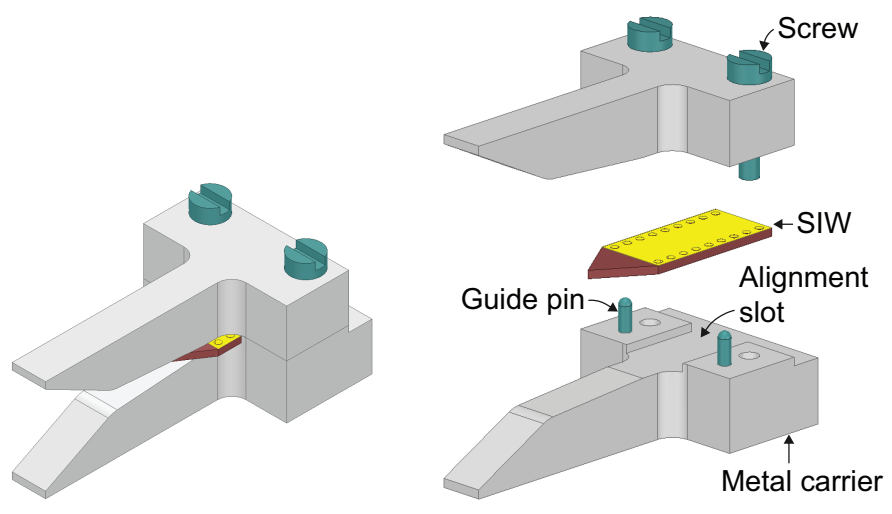

Fig. 5. Assembly of the proposed SIW-fed wideband tapered slot antenna.

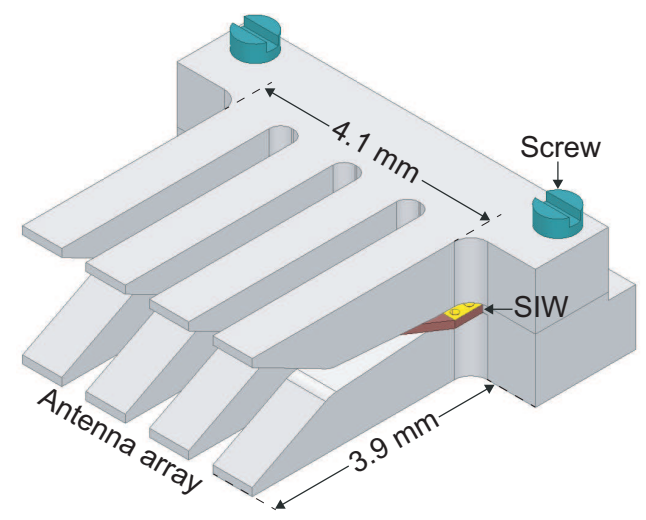

Fig. 6. A $1 \times 4$ antenna array using the proposed SIW-fed wideband tapered slot antennas.

combine the proposed wideband tapered slot antennas into an array. As is shown in Fig. 6, four identical antennas are lined up forming a $1 \times 4$ array. Depending on the applications, it can be fed by either four individual SIW inputs or a SIW power divider splitting the input into four ways. Compared with the free-space wavelength at $140 \mathrm{GHz}\left(\lambda_{0}\right)$, the proposed $1 \times 4$ array exhibits a compact structure. The occupation area is less than $4 \lambda_{0}{ }^{2}$. The total width and length of the $1 \times 4$ array are $4.1 \mathrm{~mm}$ and $3.9 \mathrm{~mm}$, respectively.

\section{Matching, Gain, And Radiation Pattern}

Fig. 7 shows the simulation results of the proposed SIW-fed wideband tapered slot antenna. The blue solid line in Fig. 7 represents the simulated return loss including the feed which remains better than $18 \mathrm{~dB}$ from $110 \mathrm{GHz}$ to $170 \mathrm{GHz}$. If the bandwidth refers to the frequency band where the return loss is better than $10 \mathrm{~dB}$, the proposed SIW-fed wideband tapered slot antenna achieves a bandwidth of $60 \mathrm{GHz}$. It covers the whole D-band and corresponds to $43 \%$ fractional bandwidth at $140 \mathrm{GHz}$. The red dashed line in Fig. 7 is the simulated antenna gain. The coordinate system for simulating the farfield radiation characteristics is assigned to the center of the antenna aperture. The maximum gain is achieved along the $x$-axis which is also the direction when $\theta$ and $\phi$ equal $90^{\circ}$ and $0^{\circ}$, respectively. A simulated gain of $11.1 \mathrm{dBi}$ is achieved

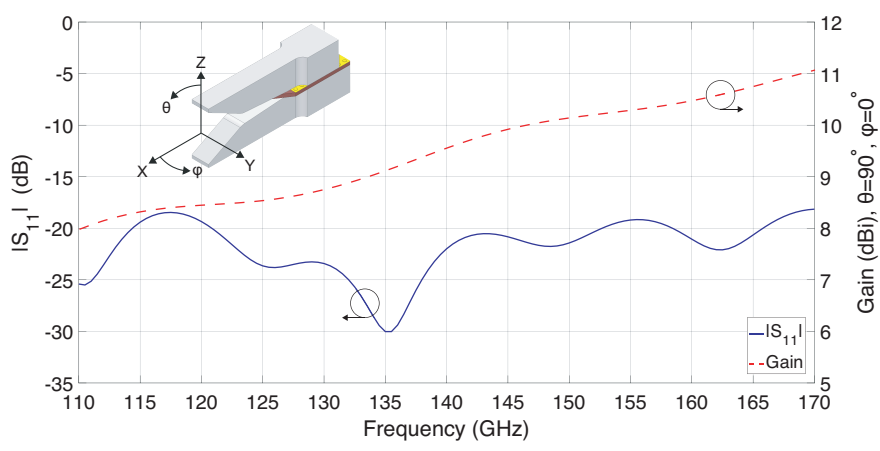

Fig. 7. Simulated return loss and gain of the SIW-fed wideband tapered slot antenna.

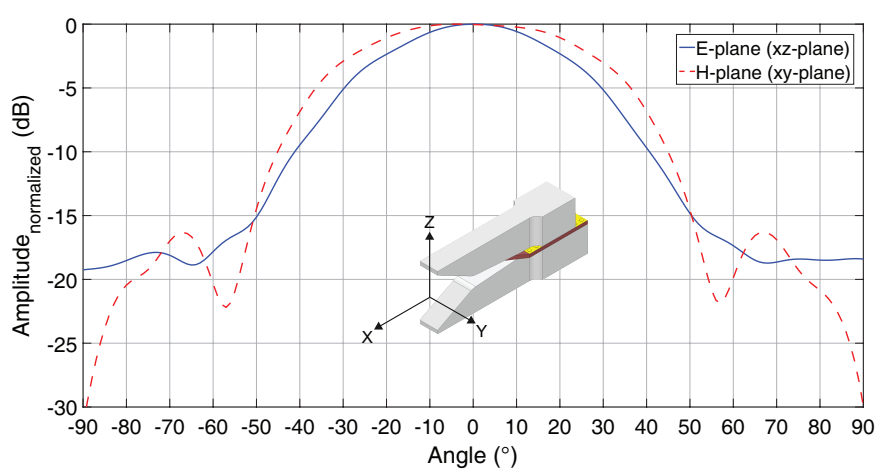

Fig. 8. Simulated far-field radiation pattern of the SIW-fed wideband tapered slot antenna at $170 \mathrm{GHz}$.

by the proposed SIW-fed wideband tapered slot antenna at $170 \mathrm{GHz}$. In addition, the antenna provides a good in-band gain uniformity since the difference between the maximum and minimum values is less than $3 \mathrm{~dB}$ at D-band.

The simulated far-field radiation pattern with normalized amplitude at $170 \mathrm{GHz}$ is shown in Fig. 8. The maximum radiation is acquired along the $x$-axis which is perpendicular to the antenna aperture. The half-power beamwidth is $46^{\circ}$ on the E-plane (xz-plane) and $60^{\circ}$ on the H-plane ( $x y$-plane).

\section{CONCLUSION}

The design of SIW-fed wideband tapered slot antenna for D-band applications has been presented. The fundamentals of the SIW and the tapered slot antenna have been explained in detail. The proposed SIW-fed wideband tapered slot antenna can provide a wideband matching and a high antenna gain at the same time. The return loss remains better than $18 \mathrm{~dB}$ from $110 \mathrm{GHz}$ to $170 \mathrm{GHz}$ and it corresponds to a bandwidth of $43 \%$ at $140 \mathrm{GHz}$. The far-field radiation pattern has been shown and an antenna gain of $11.1 \mathrm{dBi}$ has been achieved at $170 \mathrm{GHz}$ in the direction perpendicular to the antenna aperture. Besides, the in-band gain variation is less than $3 \mathrm{~dB}$ which makes the proposed SIW-fed wideband tapered slot antenna suitable for the future high-speed wireless communication systems. The assembly structure has been addressed using a compact approach and the possibility of building an antenna array has been discussed. 


\section{REFERENCES}

[1] A. Vosoogh, A. U. Zaman, and V. Vassilev, "Wideband cavity-backed slot subarray with gap waveguide feed-network for D-band applications,' in proc. 2017 11th European Conference on Antennas and Propagation (EuCAP), pp.207-209, Mar. 2017.

[2] A. Hirata et al., "120-GHz-band wireless link technologies for outdoor 10-Gbit/s data transmission," Microwave Theory and Techniques, IEEE Transactions on, vol.60, no.3, pp.881-895, Mar. 2012.

[3] Z. Shen, N. Ito, E. Sakata, C. W. Domier, N. C. Luhmann, and A. Mase, "D-band double dipole antenna for use in millimeter wave imaging systems," in proc. 2007 IEEE Antennas and Propagation Society International Symposium, pp.2658-2661, June 2007.

[4] B. Goettel, W. Winkler, A. Bhutani, F. Boes, M. Pauli, and T. Zwick, "Packaging solution for a millimeter-wave system-on-chip radar," Components, Packaging and Manufacturing Technology, IEEE Transactions on, vol.8, no.1, pp.73-81, Jan. 2018.

[5] Z. Miao et al., "140 GHz high-gain LTCC-integrated transmit-array antenna using a wideband SIW aperture-coupling phase delay structure," Antennas and Propagation, IEEE Transactions on, vol.66, no.1, pp.182190, Jan. 2018.

[6] F. F. Manzillo, A. Clemente, B. Clemente, G. Pares, A. Siligaris, and J. Luis González-Jiménez, "Transmitarray antenna with integrated frequency multiplier for high-speed D-band communications in low-cost PCB technology," in proc. 2019 13th European Conference on Antennas and Propagation (EuCAP), pp.1-4, Mar. 2019.

[7] Y. Dong, T. K. Johansen, V. Zhurbenko, and P. J. Hanberg, "A rectangular waveguide-to-coplanar waveguide transition at D-band using wideband patch antenna," in proc. 2018 48th European Microwave Conference (EuMC), pp.1045-1048, Sep. 2018.

[8] V. Vassilev et al., "Spectrum efficient D-band communication link for real-time multi-gigabit wireless transmission," in proc. 2018 IEEE/MTT-S International Microwave Symposium (IMS), pp.1523-1526, June 2018.

[9] M. Furqan, F. Ahmed, R. Feger, K. Aufinger, and A. Stelzer, "A 120-GHz wideband FMCW radar demonstrator based on a fully-integrated SiGe transceiver with antenna-in-package," in proc. 2016 IEEE MTT-S International Conference on Microwaves for Intelligent Mobility (ICMIM), pp.1-4, May 2016.

[10] M. Bertrand et al., "Substrate integrated waveguides for mm-wave functionalized silicon interposer," in proc. 2018 IEEE/MTT-S International Microwave Symposium (IMS), pp.875-878, June 2018.

[11] M. Yi et al., "Surface roughness modeling of substrate integrated waveguide in D-band," Microwave Theory and Techniques, IEEE Transactions on, vol.64, no.4, pp.1209-1216, Apr. 2016. 